\title{
Optical coherence tomography angiography of types 1 and 2 choroidal neovascularization in age-related macular degeneration during anti-VEGF therapy: evaluation of a new quantitative method
}

\author{
Henrik Faatz ${ }^{1} \cdot$ Marie-Louise Farecki $^{1} \cdot$ Kai Rothaus $^{1} \cdot$ Frederic Gunnemann $^{1} \cdot$ Matthias Gutfleisch $^{1}$. \\ Albrecht Lommatzsch ${ }^{1,2} \cdot$ Daniel Pauleikhoff ${ }^{1,2}$
}

Received: 22 August 2018 / Revised: 21 February 2019 / Accepted: 5 March 2019 / Published online: 10 April 2019

(c) The Royal College of Ophthalmologists 2019

\begin{abstract}
Purpose The aim of this pilot study was to test whether mathematical parameters of the vascular morphology of choroidal neovascularization $(\mathrm{CNV})$ can be used as biomarkers and to investigate how these parameters change during anti-vascular endothelial growth factor (VEGF) therapy.

Methods Treatment-naive CNV in exudative age-related macular degeneration (AMD) was diagnosed in 28 patients. OCTangiography (OCT-A) (Avanti/FA Optovue) performed before and after anti-VEGF therapy. The OCT-A data sets were exported to an external image processing program and vessel skeletonization was accomplished by means of edge detection. Based on this technique the total vessel length, the number of segments and the fractal dimension (FD) of the CNV were calculated before and after therapy. The results were compared with other clinical parameters such as VA and central retinal thickness (RT).

Results The total vessel length of the CNV was significantly reduced by anti-VEGF-therapy (mean value 652 pixels vs. 397 pixels; $p<0.0001$ ), as well as the number of individual vessel segments of the CNV (mean value $117 \mathrm{vs.} 76 ; p<0.0001$ ). The FD of the CNV also decreased significant reduction during therapy (mean 1.23 vs. $1.16, p<0.0001$ ). The changes in these parameters during treatment corresponded with an increase in VA and a reduction in RT.

Conclusion This pilot study demonstrates that the vascular pattern of CNV in AMD can be visualized and described using mathematical parameters of OCT-A. The changes during therapy correlate significantly with established "activity" parameters of CNV, so changes in these parameters (especially FD) may represent additional CNV "activity" biomarkers.
\end{abstract}

\section{Introduction}

For several decades, fluorescein angiography (FA) has been the gold standard for the diagnosis and characterization of neovascular age-related macular degeneration (nAMD) [1]. With the introduction of optical coherence tomography (OCT), a non-invasive imaging method became available to observe the $\mathrm{CNV}$ activity by analysing fluid distribution in the macula, other regions of the

Henrik Faatz

henrik.faatz@augen-franziskus.de

1 Department of Ophthalmology, St. Franziskus Hospital, Münster, Germany

2 Department of Ophthalmology, University of Essen-Duisburg, Duisburg, Germany retina and the retinal pigment epithelium (RPE) [2]. These two methods are used for classification of CNV by location, below the RPE (type 1, angiographically occult type) or above the RPE (type 2, angiographically classic type) [3]. Indocyanine green angiography (ICG-A) can be helpful to differentiate between additional subtypes such as idiopathic polypoidal choroidal vasculopathy (IPCV) and retinal angiomatous proliferation (type 3). Therefore, multimodal imaging has become standard for characterization of the individual type of CNV in nAMD. Early diagnosis of nAMD and recognition of "new activity" after anti-vascular endothelial growth factor (VEGF) therapy by comparison of fluid distribution in SD-OCT are crucial for maintaining or regaining the best possible visual acuity (VA) in our patients [4]. Moreover, the diagnosis is followed by invasive therapy with risks such as endophthalmitis. It is therefore important to avoid incorrect diagnoses. 
Since the introduction of non-invasive OCT angiography (OCT-A), it has been possible to detect retinal vessels in freely selected segments without superimposition. Morphological descriptions of type 1 and type $2 \mathrm{CNV}$ using OCT-A have been published [5-8].

Previous publications on activity assessment in OCT-A describe a dense vascular network at the time of initial diagnosis of $\mathrm{CNV}$, whereas $\mathrm{CNV}$ treated with anti-VEGF displays a loose vascular network or a mixed type [9]. Other authors have described a decrease in the small, thin vascular portions of CNV during anti-VEGF therapy at the segmentation level of the choriocapillaris (CC). Both type 1 and type 2 neovascularization are well-defined, distinguishable from the surrounding tissue, and significant size changes of CNV during anti-VEGF therapy can be detected [7, 10-12]. Because OCT-A is based on grey values, however, this technique opens up further "mathematical" possibilities to describe vascular patterns. This may be important for phenotypic differentiation of CNV, but may, in particular, represent a further biomarker in the analysis of "activity" parameters during anti-VEGF therapy, in addition to the established parameters such as the course of VA and the change of fluid distribution in SD-OCT.

The aim of this pilot study was to test whether mathematical parameters of the vascular morphology of CNV can be used as biomarkers and to investigate changes in these parameters during anti-VEGF therapy.

\section{Patients and methods}

The study was conducted in accordance with the tenets of the Helsinki Declaration, the local ethics committee granted its approval, and all patients gave written consent for participation in the study.

In 28 eyes of 28 patients ( 19 female, 9 male; mean age 78.3 years) the initial diagnosis of exudative AMD type 1 (10 patients), type 2 (11 patients) or mixed type (7 patients) was made by means of FA and SD-OCT (Spectralis ${ }^{\circledR}$ HRA + OCT, Heidelberg Engineering, Heidelberg, Germany). All patients were treatment naive. In addition, all patients underwent OCT-A imaging. The patients were enrolled retrospectively. All patients underwent slit-lamp microscopy and direct ophthalmoscopy via the slit-lamp in mydriasis. Additionally, OCT-A imaging was performed using AngioVue (RTVue XR Avanti; Optovue, Inc., Fremont, CA, USA). Patients with IPCV or type $3 \mathrm{CNV}$ were excluded from the study. The patients were given intravitreal anti-VEGF medications and multimodal imaging was repeated 4 weeks after the initial loading dose of three anti-VEGF injections. Patients with signs of $\mathrm{CNV}$ activity on SD-OCT at the follow-up visit were excluded.
Measurement of VA and central retinal thickness (RT) on SD-OCT and OCT-A using RTVue XR Avanti was performed before and after the loading phase of anti-VEGF treatment (three injections). The RTVue XR Avanti produces 70,000 scans every second with a wavelength of $840 \mathrm{~nm}$ and has a penetration depth of $3 \mathrm{~mm}$. The axial resolution is $5 \mu \mathrm{m}$ and the lateral resolution is $22 \mu \mathrm{m}$. The images with a size of $6 \times 6 \mathrm{~mm}$ were evaluated with the automatic segmentation of AngioVue's "choroid mode" at the level of the $\mathrm{CC}$, which has been demonstrated as "representative" of the CNV in previous studies [7, 11, 12]. In to minimize projection artefacts of the retinal vessels, a CC slab of $19 \mu \mathrm{m}$ at the level of the CC was used, as demonstrated as "characteristic" for $\mathrm{CNV}$ by Farecki et al. [7].

The image analysis method can be divided into the following steps (Fig. 1):

1. Marking of the CNV region by a physician

2. Computation of the image gradient field (Nabla operator)

3. Edge enhancement and multi-scale smoothing of the gradient field

4. Ridge/valley detection

5. Non-maximum suppression to select the skeleton pixels

6. Modelling of the vascular graph

Step 1 is realized using the software Fiji (National Institute of Mental Health, Bethesda, MD, USA). The physician exports the en-face OCTA images from the ReVUE Software and saves them loss-less as TIFF files. These images are imported to Fiji, where the CNV is interactively segmented into a polygon. Everything except the $\mathrm{CNV}$ is then removed (Fig. 1a) so that the subsequent automated MatLab program is able to analyse the CNV morphology. Steps 2 to 6 are implemented in MatLab (Mathworks, Version R2014b). The gradient field is computed (Step 2, Fig. 1b) using the Nabla operator. We achieved this by weak Gaussian smoothing followed by 1pixel finite difference of the image in the $\mathrm{x}$ and $\mathrm{y}$ directions. The edge strength is then enhanced and multi-scale smoothing is performed to propagate the edge information towards the skeleton lines (Step 3, Fig. 1c) [13]. For detection of the ridges, which correspond to the vasculature, again the Nabla operator is applied to the smoothed gradient field (Step 4, Fig. 1d). Technically, this approach corresponds to multi-scale application of the Laplace operator and the result can be interpreted as creaseness of the image [14]. Exclusion of all pixels with less than maximal creaseness leaves the skeleton pixels of the vasculature (Step 5, Fig. 1e). Finally, the skeleton pixels are grouped to form smooth vessel segments connected by nodes at bifurcations, or crossings (Step 5, Fig. 1f). 
Fig. 1 Image analysis steps of CNV OCT-A imaging

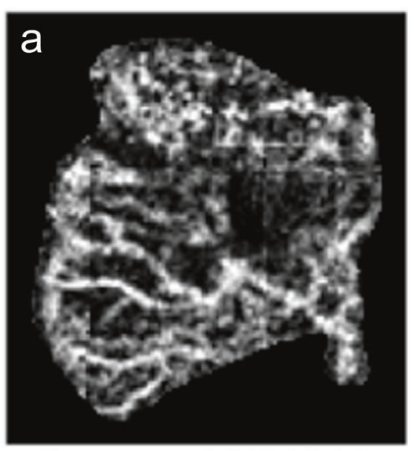

Segmented CNV field

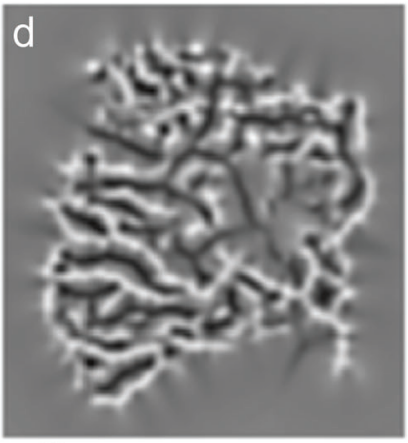

Creaseness measure

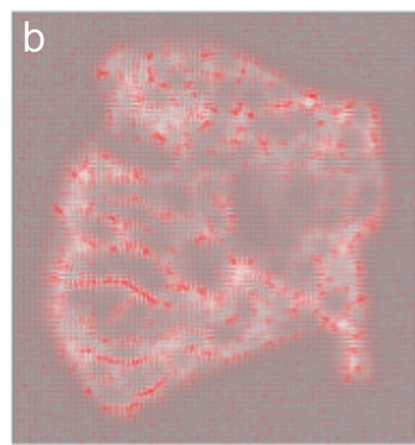

Gradient vector field

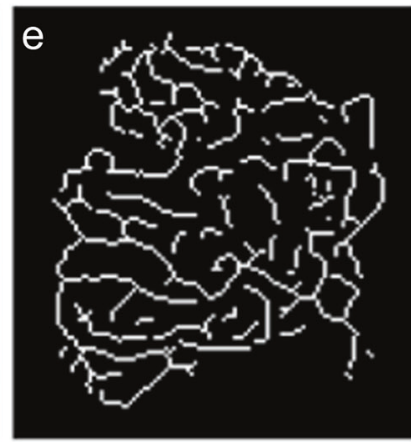

Skeleton pixel

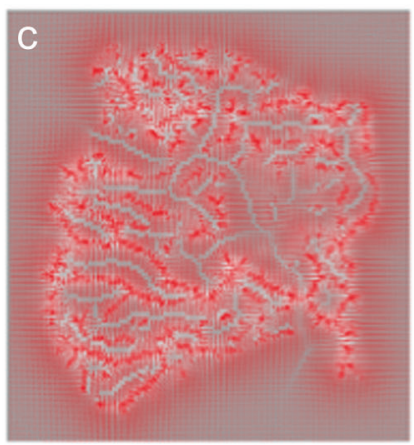

Propageted gradient vector

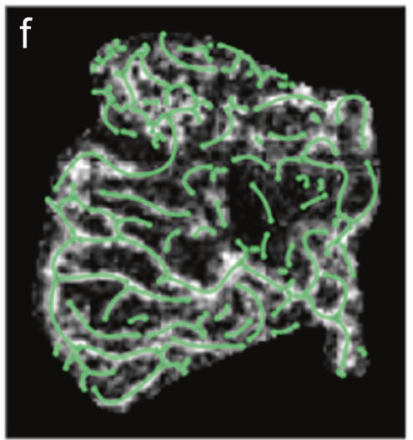

Vascular Graph

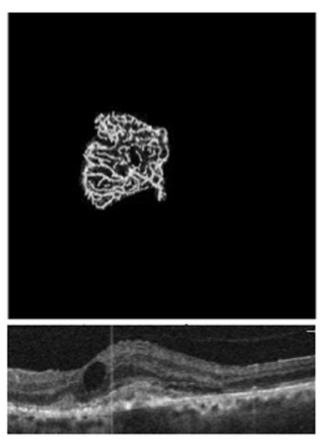

initial diagnosis

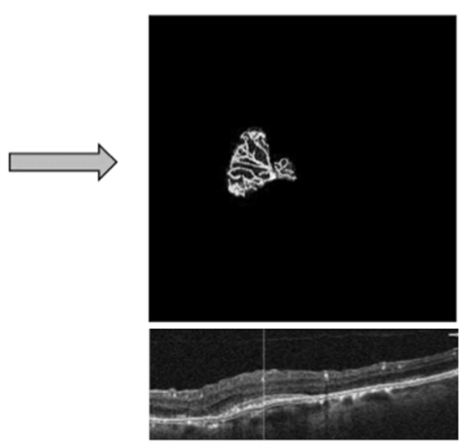

after anti-VEGF treatment

Fig. 2 Left: The vascular graph of the native $\mathrm{CNV}$ before treatment; right: after intravitreal anti-VEGF treatment Shapiro-Wilk). Therefore, we used the $t$-test for normally distributed features (such as FD) and the Wilcoxon rank-sum test for the other features (such as sum of vessel length). The level of significance was set at 5\% for all tests.

\section{Results}

VA and RT were recorded before and after treatment, and the mathematical model for the characterization and quantification of CNV was analysed before and after anti-VEGF therapy (Table 1). There was significant concordance between reduction in SD-OCT RT and reduction of the analysed vascular characteristics after treatment. However, there was no significant improvement in VA.

The mean total length of the CNV vessels (with standard deviation, SD) was 652 pixels \pm 709 SD at initial diagnosis and 397 pixels \pm 420 SD after anti-VEGF therapy; the decrease was highly significant $(p<0.0001)$ (Fig. 3).

The number of individual vascular segments of CNV was $117 \pm 115 \mathrm{SD}$ on average at initial diagnosis and $76 \pm$ 77 SD after anti-VEGF therapy, a highly significant reduction $(p<0.0001)$ (Fig. 4).

The CNV FD was also highly significantly lowered by anti-VEGF therapy, from $1.23 \pm 0.12 \mathrm{SD}$ to $1.16 \pm 0.122 \mathrm{SD}$ $(p<0.0001)$ (Fig. 5). 
Table 1 Overview of the data collected at the time of initial diagnosis (ID) and at follow-up (FU)

\begin{tabular}{|c|c|c|c|c|c|c|c|c|c|c|}
\hline & $\begin{array}{l}\text { RT } \\
{[\mu \mathrm{m}]} \\
\text { ID }\end{array}$ & $\begin{array}{l}\text { RT } \\
{[\mu \mathrm{m}]} \\
\mathrm{FU}\end{array}$ & $\begin{array}{l}\text { Vision } \\
\log \mathrm{MAR} \\
\text { ID }\end{array}$ & $\begin{array}{l}\text { Vision } \\
\text { logMAR } \\
\text { FU }\end{array}$ & FD ID & FD FU & $\begin{array}{l}\text { Vessel } \\
\text { segments } \\
\text { ID }\end{array}$ & $\begin{array}{l}\text { Vessel } \\
\text { segments } \\
\text { FU }\end{array}$ & $\begin{array}{l}\text { Vessel } \\
\text { length } \\
{[\mathrm{mm}]} \\
\text { ID }\end{array}$ & $\begin{array}{l}\text { Vessel } \\
\text { length } \\
{[\mathrm{mm}]} \\
\text { FU }\end{array}$ \\
\hline Median & 380 & 279 & 0.3 & 0.25 & 1.220 & 1.155 & 64 & 45 & 7.04 & 5.75 \\
\hline Mean & 384 & 289 & 0.38 & 0.33 & 1.225 & 1.162 & 117 & 76 & 13.04 & 7.93 \\
\hline SD & 90 & 59 & 0.32 & 0.31 & 0.124 & 0.122 & 115 & 77 & 14.17 & 8.41 \\
\hline Max & 636 & 438 & 1.3 & 1.3 & 1.460 & 1.387 & 421 & 273 & 8.4 & 5.5 \\
\hline Min & 251 & 208 & 0.1 & 0 & 0.987 & 0.938 & 13 & 12 & 0.3 & 0.2 \\
\hline$p$-value & $<0.0001$ & & 0.09 & & $<0.0001$ & & $<0.0001$ & & $<0.0001$ & \\
\hline
\end{tabular}

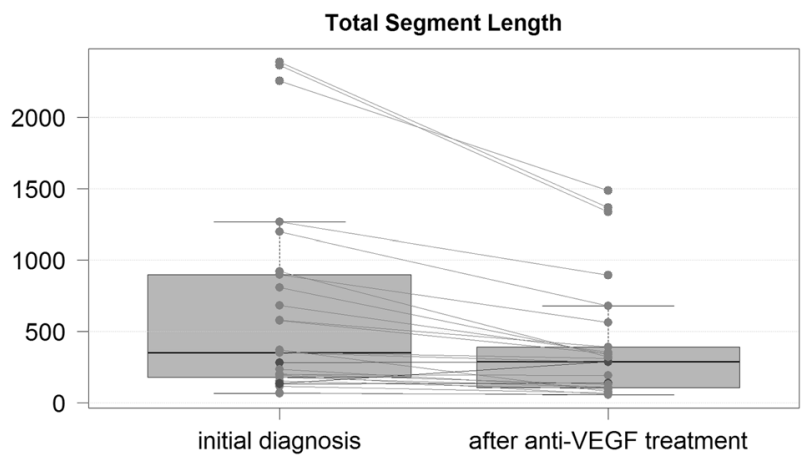

Fig. 3 The mean total length of CNV vessels at initial diagnosis and after anti-VEGF treatment. The reduction is highly significant $(p<$ $0.0001)$

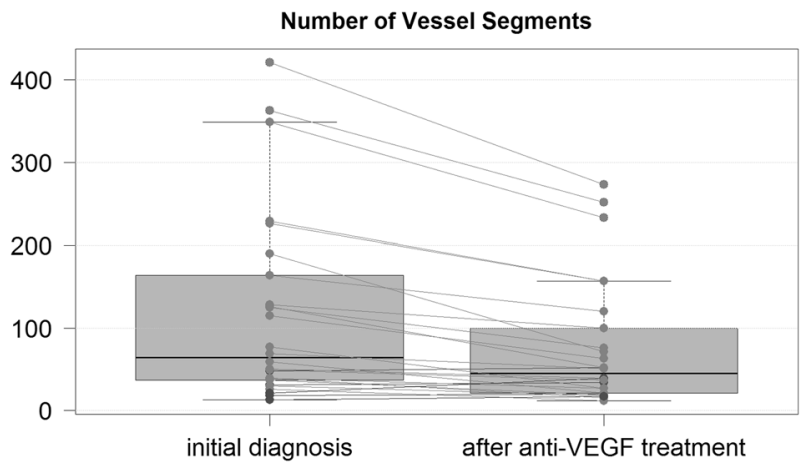

Fig. 4 The number of individual vascular segments of CNV at initial diagnosis and after anti-VEGF treatment. The reduction is highly significant $(p<0.0001)$

\section{Discussion}

The direct and detailed examination of CNV in nAMD by OCT-A imaging yields a valuable information gain. However, more data from multimodal imaging with SD-OCT and FA are still required to enable classification of the findings. Because OCT-A techniques are based on grey value analysis, a mathematical approach to the description and characterization vessels appears useful. In addition, the

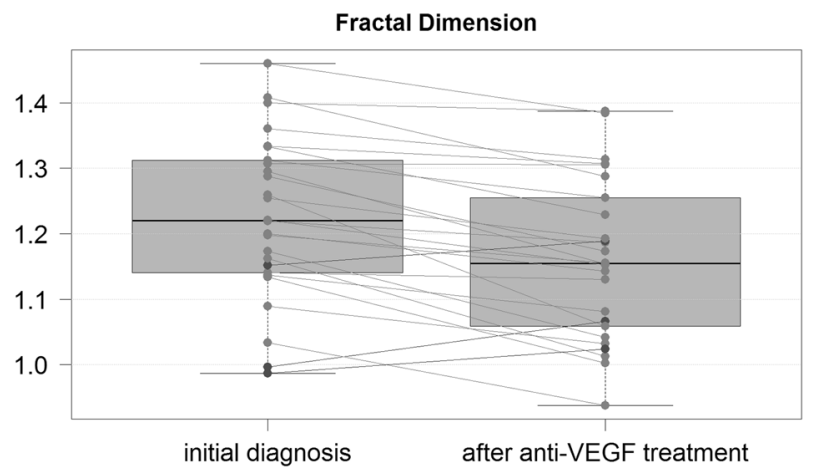

Fig. 5 The CNV FD at initial diagnosis and after anti-VEGF treatment. The reduction is highly significant $(p<0.0001)$

parameters quantified by this new technique may emerge as a further activity criterion of CNV alongside the established fluid distribution analysis in SD-OCT for observation and evaluation of the changes during ant-VEGF therapy. The present pilot study addressed both aspects.

However, one major limitation of the OCT-A technique with regard to $\mathrm{CNV}$ changes during anti-VEGF therapy has to be recognized. The "visualization" in OCT-A relates to changes of flow within a specific window between two OCT scans. Therefore, if the flow is slow, a "vessel" will not be recognized. Because of this, reduction of flow does not directly imply a decrease in CNV size. Bearing these limitations in mind, the development of a mathematical model for the characterization and differentiated categorization of CNV has two important aspects. Previous work has shown that the detection of CNV with OCT-A imaging has good sensitivity of $86 \%$ and specificity of $82 \%$, independent of the examiner's experience. In this study, OCT-A was even superior to FA, which had sensitivity of only $75 \%$ and specificity of $82 \%$ [15]. Another study that supported our findings found sensitivity of $87 \%$ and specificity of $68 \%$ for the representation of CNV using OCT-A (LIT). It has already been shown that silent $\mathrm{CNV}$ can be detected using OCT-A, whereas SD-OCT and FA do not detect silent $\mathrm{CNV}$, but signs of activity, such as subretinal and/or intraretinal fluid or leakage, are not seen on OCT-A [16]. At 
present, therefore, SD-OCT or FA is necessary to assess CNV activity. These findings currently have no consequences for treatment, but it is likely that the interpretation of vascular changes over the course of time will help to identify high-risk patients who develop nAMD and enable treatment of such patients at an early stage. Therefore, the first aspect of the assessment of a mathematical model for the description of CNV would be FA-independent differentiation of $\mathrm{CNV}$ using specific vascular characteristics rather than dye distribution and leakage.

The second aspect of mathematical characterization of vascular pattern would be, that treatment effects may be described in ways other than the established analysis of fluid distribution in SD-OCT. In CNV, pathological vessels from the CC grow below the RPE or underneath the retina, potentially causing subretinal and/or intraretinal fluid leakage. SD-OCT is used for therapy monitoring, analysing the fluid distribution, the presence of subretinal fluid, or the increase in intraretinal fluid, which represent activity criteria and indications for treatment. In this assessment only indirect conclusions are drawn from changes in CNV. The decrease in several mathematical parameters during antiVEGF therapy as observed in this pilot study may therefore indicate the possibility of using this approach as a second parameter for "CNV activity". Especially the total vessel length of CNV decreases significantly during therapy. However, the number of individual vascular segments of a CNV was also significantly reduced by the treatment. This is in accordance with the findings of previous studies that especially a reduction of peripheral small capillary vascular segments of a CNV can be observed during therapy, but that the larger central vascular segments are retained [10, 17]. This fact was interpreted to result from higher sensitivity of capillaries than mature vessels by virtue of their lack of pericytes, making them particularly amenable to anti-VEGF therapy. We also used the FD as a further parameter for objective description of the complexity of the vascular pattern of the CNV. The FD is calculated from the number of pixels involved in the CNV image and can assume values between 0 and 2 . It describes the complexity of the vascular structure and increases with rising complexity. We saw a significant reduction in FD from the initial analysis of $\mathrm{CNV}$ to the analysis after the loading phase of anti-VEGF therapy. This shows that the vascular structure of CNV becomes "looser", i.e. there is a decrease in vascular segments and branches. Al-Sheikh et al. also compared the FD of active CNV against inactive CNV in exudative AMD and found a significant reduction in FD in inactive CNV, consistent with our findings [18, 19].

Therefore, we can conclude that description and characterization of CNV using "characteristic" CC slabs and analysis of mathematical parameters such as total vessel length, number of vessel segments and FD is feasible both initially and during anti-VEGF therapy and that comparison shows a therapeutic reduction of the CNV.

However, our pilot study certainly exhibits limitations. First, the study population was small and the CNV had to be marked manually by the investigator. The accuracy achieved depends on the investigator's experience with OCT-A imaging and on the image resolution, which is limited by the technical development of the manufacturers. The agreement of different assessors of OCT-A findings in CNV is comparable to that for FA [20]. Second, high image quality is crucial for any further mathematical analysis. This has already improved considerablywith the development of eye-tracking systems to prevent motion artefacts in the images. Projection artefacts also limit the detection accuracy and quantification of $\mathrm{CNV}$. In addition, segmentation may change over time, as retinal morphology is usually altered considerably by the loss of intra- and/or subretinal fluid, and thus the segmentation levels generated by the manufacturer may also change. However, further technical improvements like artefact removal and new developments such as swept-source technology, which uses a longer wavelength of $1050 \mathrm{~nm}$ and penetrates deeper into the choroid, will improve the resolution of CNV imaging. In addition, future advances in OCT-A imaging and mathematical analysis should address the three-dimensional pathology of CNV. Furthermore, analysis of the findings regarding the "mathematical" parameters in this pilot study shows that although the described reduction was visible in the majority of the CNV, some CNV consistently demonstrated an opposite effect. This could be a sign of inadequate sensitivity of the method, but may also be an indication of the existence of different "types" of initial CNV or of differing behaviour of CNV during anti-VEGF therapy. These aspects have to be evaluated in further studies.

In summary, our pilot study demonstrates that the vascular pattern of CNV in AMD can be visualized and described using mathematical parameters of OCT-A. The changes during therapy correlate significantly with established "activity" parameters of CNV, so changes in these mathematical parameters (especially FD) may represent additional biomarkers of CNV "activity". Further prospective and longitudinal studies are needed to clarify the clinical indication for the "mathematical" characterization of $\mathrm{CNV}$ in clinical practice.

\section{Summary}

\section{What was known before}

- Fluorescein angiography (FA) and optical coherence tomography (OCT) has been the gold standard for the diagnosis, to observe the CNV activity and 
characterization of neovascular age-related macular degeneration. Since the introduction of non-invasive OCT angiography (OCT-A), it has been possible to detect retinal vessels in freely selected segments without superimposition. Morphological descriptions of type 1 and type $2 \mathrm{CNV}$ using OCT-A have been published.

\section{What this study adds}

- We demonstrates that the vascular pattern of CNV in AMD can be visualized and described using mathematical parameters of OCT-A. The total vessel length, the number of segments and the fractal dimension (FD) of the CNV were calculated and the changes during therapy correlate significantly with established "activity" parameters of CNV.

\section{Compliance with ethical standards}

Conflict of interest The authors declare that they have no conflict of interest.

Publisher's note: Springer Nature remains neutral with regard to jurisdictional claims in published maps and institutional affiliations.

\section{References}

1. Bressler NM. Treatment of Age-Related Macular Degeneration with Photodynamic Therapy (TAP) Study Group. Photodynamic therapy of subfoveal choroidal neovascularization in age-related macular degeneration with verteporfin: two-year results of 2 randomized clinical trials-tap report 2. Arch Ophthalmol Chic Ill 1960. 2001;119:198-207.

2. Brown DM, Kaiser PK, Michels M, Soubrane G, Heier JS, Kim $\mathrm{RY}$, et al. Ranibizumab versus verteporfin for neovascular agerelated macular degeneration. N Engl J Med. 2006;355:1432-44. https://doi.org/10.1056/NEJMoa062655.

3. Freund KB, Zweifel SA, Engelbert M. Do we need a new classification for choroidal neovascularization in age-related macular degeneration? Retina Phila Pa. 2010;30:1333-49. https://doi.org/ 10.1097/IAE.0b013e3181e7976b.

4. Matthé E, Sandner D. Early treatment of exudative age-related macular degeneration with ranibizumab (Lucentis ${ }^{\circledR}$ ): the key to success. Ophthalmol Z Dtsch Ophthalmol Ges. 2011;108:237-43. https://doi.org/10.1007/s00347-010-2282-9.

5. Kuehlewein L, Bansal M, Lenis TL, Iafe NA, Sadda SR, Bonini Filho MA, et al. Optical coherence tomography angiography of type 1 neovascularization in age-related macular degeneration. Am J Ophthalmol. 2015;160:739-.e2. https://doi.org/10.1016/j. ajo.2015.06.030

6. El Ameen A, Cohen SY, Semoun O, Miere A, Srour M, QuarantaEl Maft M, et al. Type 2 neovascularization secondary to age-related macular degeneration imaged by optical coherence tomography angiography. Retina Phila Pa. 2015;35:2212-8. https://doi.org/10. 1097/IAE.0000000000000773.
7. Farecki M-L, Gutfleisch M, Faatz H, Rothaus K, Heimes B, Spital $\mathrm{G}$, et al. Characteristics of type 1 and $2 \mathrm{CNV}$ in exudative AMD in OCT-Angiography. Graefes Arch Clin Exp Ophthalmol. 2017:1-9. https://doi.org/10.1007/s00417-017-3588-y.

8. Coscas G, Lupidi M, Coscas F, Français C, Cagini C, Souied EH. Optical coherence tomography angiography during follow-up: qualitative and quantitative analysis of mixed type I and II choroidal neovascularization after vascular endothelial growth factor trap therapy. Ophthalmic Res. 2015;54:57-63. https://doi.org/10. 1159/000433547.

9. Sulzbacher F, Pollreisz A, Kaider A, Kickinger S, Sacu S, Schmidt-Erfurth U. Identification and clinical role of choroidal neovascularization characteristics based on optical coherence tomography angiography. Acta Ophthalmol. 2017. https://doi.org/ 10.1111/aos.13364.

10. Spaide RF. Optical coherence tomography angiography signs of vascular abnormalization with antiangiogenic therapy for choroidal neovascularization. Am J Ophthalmol. 2015. https://doi.org/ 10.1016/j.ajo.2015.04.012.

11. Costanzo E, Miere A, Querques G, Capuano V, Jung C, Souied EH. Type 1 choroidal neovascularization lesion size: indocyanine green angiography versus optical coherence tomography angiography. Invest Ophthalmol Vis Sci. 2016;57:307-13. https://doi. org/10.1167/iovs.15-18830.

12. Faatz H, Rothaus K, Gunnemann F, Gutfleisch M, HeimesBussmann B, Lommatzsch A, et al. Changes in OCT Angiography of Type $2 \mathrm{CNV}$ in Neovascular AMD during Anti-VEGF Treatment. Klin Monatsbl Augenheilkd. 2017;234:1125-31. https://doi. org/10.1055/s-0043-118346.

13. Rothaus K, Jiang X. Multi-scale midline extraction using creaseness. In: Pattern recognition and image analysis. Lecture Notes in Computer Science. Springer, Berlin, Heidelberg; 2005:502-11. https://doi.org/10.1007/11552499_56.

14. López AM, Lloret D, Serrat J, Villanueva JJ. Multilocal creaseness based on the level-set extrinsic curvature. Comput Vis Image Underst. 2000;77:111-44. https://doi.org/10.1006/cviu. 1999.0812 .

15. Souedan V, Souied EH, Caillaux V, Miere A, Ameen AE, BlancoGaravito R. Sensitivity and specificity of optical coherence tomography angiography (OCT-A) for detection of choroidal neovascularization in real-life practice and varying retinal expertise level. Int Ophthalmol. 2017. https://doi.org/10.1007/s10792017-0559-6

16. Palejwala NV, Jia Y, Gao SS, Liu L, Flaxel CJ, Hwang TS, et al. Detection of nonexudative choroidal neovascularization agerelated macular degeneration with optical coherence tomography angiography: Retina. 2015;35:2204-11. https://doi.org/10.1097/ IAE.0000000000000867.

17. Kuehlewein L, Sadda SR, Sarraf D. OCT angiography and sequential quantitative analysis of type 2 neovascularization after ranibizumab therapy. Eye Lond Engl. 2015;29:932-5. https://doi. org/10.1038/eye.2015.80.

18. Al-Sheikh M, Iafe NA, Phasukkijwatana N, Sadda SR, Sarraf D. Biomarkers of Neovascular activity in age-related macular degeneration using oct angiography. Retina Phila Pa.2017; https:// doi.org/10.1097/IAE.0000000000001628.

19. Lumbroso B, Rispoli M, Savastano MC. Longitudinal optical coherence tomography-angiography study of type 2 naive choroidal neovascularization early response after treatment: Retina. 2015;35:2242-51. https://doi.org/10.1097/IAE.0000000000000879.

20. Lindner M, Fang PP, Steinberg JS, Domdei N, Pfau M, Krohne TU, et al. OCT angiography-based detection and quantification of the neovascular network in exudative AMD. Invest Ophthalmol Vis Sci. 2016;57:6342-8. https://doi.org/10.1167/iovs.16-19741. 